\title{
What's app? Electronic health technology in inflammatory bowel disease
}

\author{
Alissa Walsh, Simon Travis \\ Translational Gastroenterology Unit, John Radcliffe Hospital, Oxford, UK
}

Electronic health (eHealth) data collection is increasingly used in many chronic illnesses, to track pattern of disease. eHealth systems have the potential to revolutionize care. Inflammatory bowel disease (IBD) is a paradigm for such an approach: this is a chronic disease that usually affects young and technologically literate patient population, who are motivated to be involved in their own care. A range of eHealth technologies are available for IBD. This review considers the strengths and weaknesses of 7 platforms that focus on patient-provider interaction. These have been developed in Denmark, United States, the Netherlands, and the United Kingdom, demonstrating an international interest in this form of technology and interaction. Not only do these technologies aim to improve care but they also have the potential to collect large amounts of information. Information includes demographics and patient reported outcomes (symptoms, quality of life), quality of care (steroid use, among other metrics) and outcomes such as hospitalization. These data could inform quality improvement programmes to improve their focus. eHealth technology is also open to machine learning to analyze large data sets, through which personalized algorithms may be developed. (Intest Res 2018;16:366-373)

Key Words: Mobile applications; Electronic health records; Inflammatory bowel disease

\section{INTRODUCTION}

Conventional monitoring and management of patients with IBD depends on assessment during brief clinic visits several weeks or many months apart. These visits are often scheduled in advance, but given the unpredictable nature of the disease, flares are often discordant from scheduled visits. This does not seem to be the best way of understanding the impact of relapsing and remitting inflammatory disease. Furthermore, the global increasing incidence of IBD, ${ }^{1}$ will compound the strain on health care resources.

Tele-medicine, digital data collection or electronic health (eHealth), simply mean that electronic devices (such as smartphones, tablets, or lap-tops) are used to collect information.

Received March 13, 2018. Revised May 9, 2018.

Accepted May 10, 2018. Published online July 11, 2018

Correspondence to Alissa Walsh, Translational Gastroenterology Unit, John

Radcliffe Hospital, Headley Way, Oxford OX3 9DU, UK. Tel: +44-7495920267,

E-mail: alissa.walsh@ndm.ox.ac.uk
eHealth technologies will revolutionize care delivery and patient engagement in many chronic diseases. By using technology, patients can participate in their own care by signaling health outcomes during year round monitoring to enable earlier detection and treatment of exacerbations of disease activity. ${ }^{2}$ Web-based management systems have been created for chronic diseases such as asthma, ${ }^{3}$ diabetes mellitus, ${ }^{4,5}$ congestive heart failure, ${ }^{6,7}$ hypertension ${ }^{8}$ and anticoagulation. ${ }^{9}$

IBD is a paradigm for such an approach: this is a chronic disease that usually affects young and technologically literate patient population, who are motivated to be involved in their own care. A survey of over 5,000 patients in 6 European countries and Canada showed that $88 \%$ search online for information about IBD, most doing so several times a month (IBD 2020 survey www.IBD2020.org, presented to the European Parliament 17 October 2013). Two-thirds would be interested in mobile technology to help manage their IBD. Involving patients in real-time, digital data entry and measurement of their own outcomes to which both patient

๑ Copyright 2018. Korean Association for the Study of Intestinal Diseases. All rights reserved.

This is an Open Access article distributed under the terms of the Creative Commons Attribution Non-Commercial License (http://creativecommons.org/licenses/by-nc/4.0)

which permits unrestricted non-commercial use, distribution, and reproduction in any medium, provided the original work is properly cited. 
and health care teams have access, is a way to empower the patient and offer cost-effective solutions to increasing health care demand.

The simple addition of patient-reported outcomes (PROs) to best conventional care has been shown to improve mortality in patients with metastatic malignancy at the Sloane Kettering Memorial Hospital. ${ }^{10}$ The same appears to apply to improving outcomes in the management of chronic diseases. ${ }^{11,12}$ For IBD, through the International Consortium of Health Outcomes Measurement (ICHOM), PROs have been agreed between patients, patient associations and multidisciplinary specialists. ${ }^{13}$

eHealth technology has the potential to collect large amounts of information including demographics, patient reported outcome measures (PROMs, most notably quality of life $[\mathrm{QoL}])$ and quality of care (QoC) parameters. If patient information is entered electronically by the patient, data quality is improved and more complete than conventional paper-pencil versions of the same instrument. ${ }^{14}$ Electronic PROMs also enable a reduction in human resources, making routine data collection feasible in busy clinical practice. ${ }^{15-18}$ Because eHealth is a rapidly evolving field, this article aims to summarize IBD eHealth programmes in current practice.

\section{eHealth TECHNOLOGY IN IBD}

A range of eHealth technologies are available in IBD. A critical distinction is whether or not that technology is directly connected to patient care. Many apps can be downloaded onto a mobile device by a patient with IBD, but these are largely personal data collection diaries to track symptoms, log meals or manage medications, since they do not connect the data with the patient's clinical record. There is thus no nexus between the eHealth technology and care, even if the information is provided to the clinician as part of an outpatient visit.

A study from Melbourne systematically assessed eHealth technologies targeted at patients. Through searches of app stores, 26 out of 238 products found were relevant to patients with IBD. Around half of these (54\%) had diary functionality and a third (39\%) provided health information, but only $19 \%$ had medical involvement in their design. ${ }^{19}$ None offered decision-support to facilitate the self-initiation of medical therapy.

It appears clear that health system quality programmes need patient-provider interaction to achieve improvements in care. ${ }^{20,21}$ Consequently, stand-alone technologies need to be distinguished from those where the information entered by patients is intended to operate as a component of clinical care and is accessible to their treating team.

A literature search for eHealth technologies that focus on patient-provider interaction in IBD identified the following:

- Constant-Care ${ }^{22}$

- UC-HAT ${ }^{23,24}$

- HealthPROMISE ${ }^{25}$

- myIBDcoach $^{26}$

- UCLA eIBD ${ }^{27}$

- IBD Qorus ${ }^{28,29}$

- TrueColours Ulcerative Colitis ${ }^{30}$

Table $1^{22,23,30-35}$ gives an overview of eHealth tools for IBD that involve patient-provider interaction, with some information about each programme and those currently in development.

\section{Constant-Care}

Constant-Care (www.constant-care.dk) is a Danish eHealth technology originally developed in 2009 for patients with mild to moderate UC. ${ }^{22}$ In the event of relapse, patients were requested to $\log$ on daily and complete the Simple Colitis Clinical Activity Index (SCCAI). Once remission was achieved, patients were asked to use the programme monthly until the next relapse occurred. When patients entered their symptoms online, their status appeared as a simple traffic light: red indicated highly active UC (SCCAI $\geq 5$ ), yellow moderately active UC (SCCAI 2-4), and green quiescent disease ( $\mathrm{SCCAI} \leq 1)$. This status was supplemented with the SCCAI graph, which was available to both the patient and physician. QoL, as measured by the Short Inflammatory Bowel Disease Questionnaire (SIBDQ) was also entered at the beginning and end of each relapse. Treatment with oral $4 \mathrm{~g}$ mesalazine (5-aminosalicylic acid, 5-ASA) was recommended by the programme if a relapse occurred. When patients entered remission, the system recommended a maintenance dose, usually half that of the induction dose. Routine visits continued as usual, but web-patients were informed that they could cancel their appointments if they felt secure.

A randomized trial was performed in Denmark and Ireland whereby Constant-Care was compared (1:1) with a control group who continued conventional care in the IBD outpatient clinic, as well as with 106 patients in a historical cohort group. A total of 333 patients (233 Denmark, 100 Ireland) were randomized. Eighty-eight percent of patients reported that they preferred this approach to conventional care. Seventy-seven percent of those in the control group expressed interest in using Constant-Care. ${ }^{36}$ 


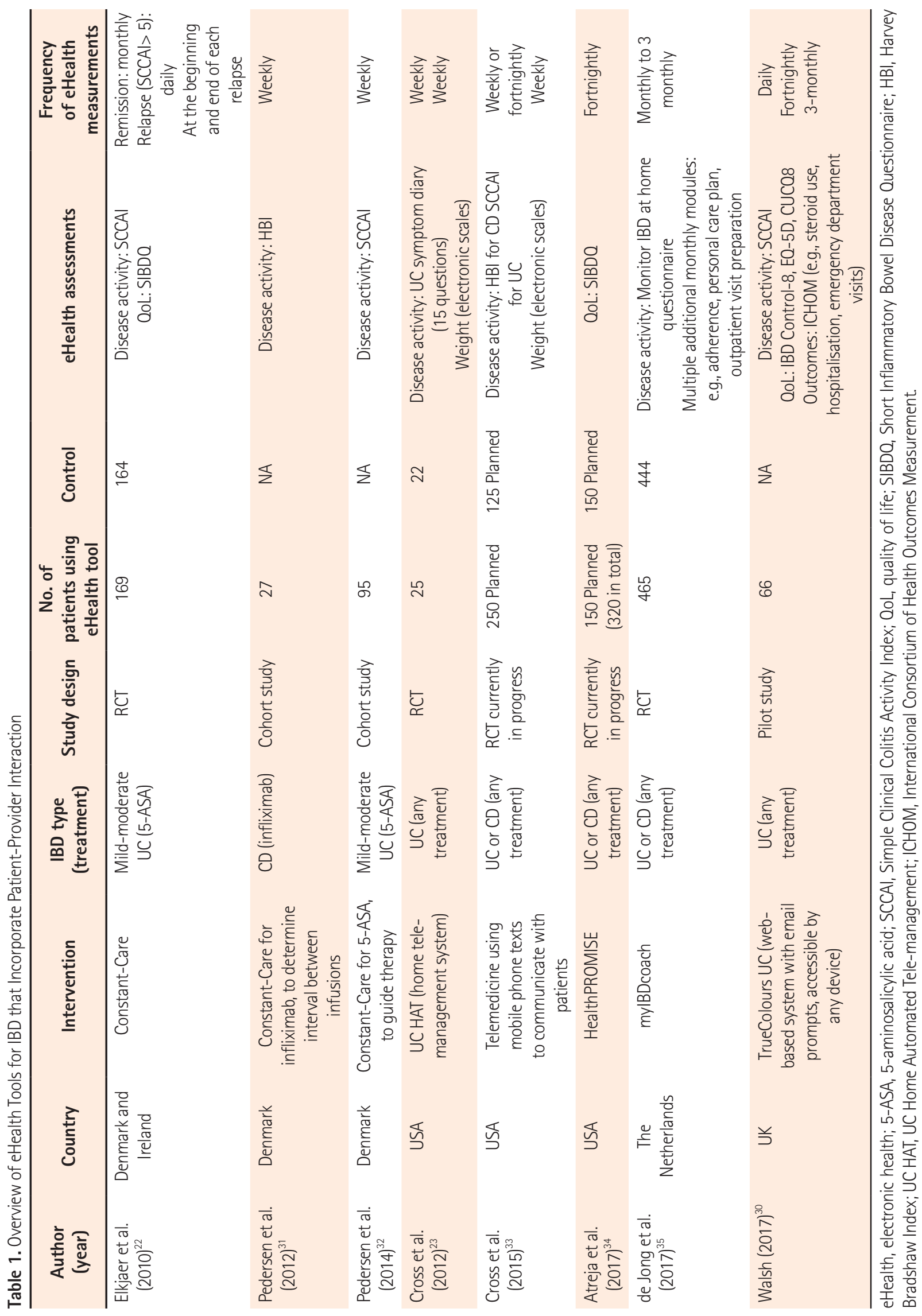


No significant difference in disease activity, a secondary outcome of the study, based on the SCCAI, was found between web-based and control groups at 12 months (OR, $2.74 ; P=\mathrm{NS})$. However, adherence to 4 weeks' acute treatment was increased by $31 \%$ in Denmark and $44 \%$ in Ireland, compared to control groups. Relapses in the web-based group were of shorter duration than in the controls both in Denmark (median, 18 days; 95\% CI, 10-21 days vs. median, 77 days; 95\% CI, 46-108 days, $P<0.001$ ) and in Ireland (median 30 days; $95 \%$ CI, 2-37 days vs. median, 70 days; $95 \%$ CI, $7-217$ days, $P<0.03)$. At the time of relapse, $100 \%$ of the Constant-Care patients in Denmark started treatment with high dose oral 5-ASA, compared to $10 \%$ of control patients $(P<0.001)$. In Ireland only $15 \%$ of Constant-Care patients started treatment with high dose 5-ASA compared to $10 \%$ controls.

Steroid use and hospitalization were similar between the groups and there was no difference in adverse events due to 5-ASA treatment. In Denmark, both acute visits and routine visits were significantly reduced in the Constant-Care group compared to the control group (21 acute visits vs. 107 acute visits and 35 routine visits vs. 92 routine visits, respectively, $P<0.001)$. The financial saving to the department was 189 euros/patient/year. Contact via e-mail and phone calls were higher in the Constant-Care group (107 vs. 24, episodes of contact in controls), which off sets the outpatient savings. ${ }^{31}$

In 2012, Constant-Care was used to individualize infliximab dosing in patients with CD. Disease activity was assessed by weekly electronic entry of the Harvey Bradshaw Index (HBI). This was combined with a fecal calprotectin resulted (stool sample mailed to hospital and result entered into the programme by investigators). An unvalidated Inflammatory Burden (IB) score was derived by combining the HBI and the fecal calprotectin results. This result categorized patients into green (IB $\leq 5$ ), amber (IB 6-7) or red (IB $\geq 8$ ) and instructions were given accordingly. Paper questionnaires for the SIBDQ were completed at 0, 6 and 12 months with no significant difference reported between baseline and 12 months ( 55 vs. $55, P=0.74$ ). ${ }^{31}$

In 2014, Pedersen et al. ${ }^{32}$ used the Constant-Care platform to perform a prospective, open-label, web-guided study of 3 months' mesalazine therapy for 95 patients with mild to moderately active UC. SCCAI was entered on a weekly basis and fecal calprotectin result was once again entered by the investigators. An IB score was derived from these 2 results and web-based advice was given. Eighty-six percent of patients were adherent to the web therapy, with a significant reduction in mean symptom scores (SCCAI; 4.6 vs. $1.6, P<0.001)$ and mean fecal calprotectin $(437 \mathrm{mcg} / \mathrm{g}$ vs. $195 \mathrm{mcg} / \mathrm{g}, P<0.001)$ at week 0 and week 12 respectively. Almost $90 \%$ of patients were able to decrease their dose of mesalazine by week 12 of the study. In spite of these promising results, however, Constant-Care is not widely used even in Denmark, probably because of the amount of healthcare professional support needed to make it useful to patients.

\section{UC HAT}

The UC Home Automated Tele-management trial (UC HAT) randomized 47 patients to UC HAT $(\mathrm{n}=25)$ or conventional care $(\mathrm{n}=22)$ for 12 months. UC HAT patients answered questions regarding disease activity (unvalidated UC symptom diary: 15 questions decided on by study group, unvalidated) and weight (as a surrogate for nutritional status) on a weekly basis. An educational curriculum was delivered after each session. Alert thresholds and action plans were generated by the treating physician at registration. Conventional care was defined as routine follow-up, written action plans and educational fact sheets. It was reported that there were no significant differences in disease activity or QoL (as judged by the 15 questions asked) between the 2 groups after 12 months and more than a third of patients withdrew from the study. ${ }^{23}$

Nevertheless, a modified version it is currently being assessed in a multicenter randomized controlled trial comparing conventional care to a telemedicine intervention that uses mobile phones and a secure provider portal to monitor symptoms, side effects, medications and weight on a weekly or fortnightly basis. Patients will record clinical activity (SCCAI if UC and HBI if CD). The primary analysis will compare change in disease activity (Seo index for UC and CDAI for CD) and disease-specific QoL scores (IBDQ). Secondary outcomes, including health care utilization (hospitalization, emergency department visits, prescriptions, general practitioner visits), general QoL (Short-Form 36), patient knowledge and system satisfaction (Client-Satisfaction Questionnaire) will be compared among the groups. ${ }^{33}$ The system is interactive, but depends on individualized specialist input from healthcare professionals, making it resource intensive. Unlike Constant-Care, UC HAT still has to demonstrate a positive impact on disease activity, QoL, or medication adherence, but the system appears open to artificial intelligence (AI) machine learning, which may allow future development of automated personalized responses. 


\section{HealthPROMISE}

HealthPROMISE is a unique cloud-based PRO and decision tool developed at Sinai AppLab, Icahn School of Medicine at Mount Sinai, New York. The app has been implemented at 7 IBD centers and a single center randomized controlled trial is currently in progress. The aim is to evaluate whether patient-centered self-monitoring and a collaborative decision making platform will improve QoL (measured by the SIBDQ). For those randomized to HealthPROMISE (162/320), as opposed to an educational app, data are entered every 2 weeks and these data are visible to the health provider through their hospital electronic patient record. Both QoL and QoC have been incorporated into HealthPROMISE, allowing longitudinal measurements over a period of time.

Baseline assessment have shown that fatigue and tension (anxiety) were the 2 most important drivers of poor QoL. One year interim results show that $75 \%$ of patients continue to login to the HealthPROMISE app every fortnight. During a median follow-up of 495 days, the proportion of patients meeting all eligible QoC parameters significantly increased in the intervention group compared to the control group using the educational app (increase of $28 \%$ vs. $9 \%, P<0.01$ ). Overall QoL started to improve among those randomized to HealthPROMISE within 5 months and was consistently above the control arm through a median 18 months (495 days). ${ }^{34}$ The responsiveness of the system is contingent on direct contact with the patient's IBD specialist team.

\section{4. myIBDcoach}

Dutch investigators have developed myIBDcoach, a telemedicine system for patients with IBD, regardless of phenotype, severity, or treatment. ${ }^{26}$ It monitors disease activity monthly using the unvalidated Monitor IBD at Home questionnaire. It also has multiple monthly modules including medication adherence and personal care plans and provides e-learning opportunities. Initially, 30 consecutive outpatients from 3 hospitals trialed the system for 3 months. Patients graded system satisfaction (Likert scale 0 -10) with a mean of 7.8 and $93 \%$ reported that they would recommend mylBDcoach to other patients.

Over an 8-month period in 2015, 909 patients were randomized (1:1) to use myIBDcoach (intervention group) or conventional care (control group) and were followed for 12 months. Patients using myIBDcoach were invited to visit the outpatient clinic at least once per year, or as needed if they felt a visit unnecessary.

The mean $(+\mathrm{SD})$ number of outpatient visits during a flare was lower in the myIBDcoach group compared to controls ( $1.55 \pm 1.50$ vs. $2.34 \pm 1.64 ; P<0.001)$. The mean number of hospitalizations was also lower in the myIBDcoach group compared to controls $(0.05 \pm 0.28$ and $0.10 \pm 0.54 ; P<0.001)$. No differences were observed in number of flares, corticosteroid use, emergency visits, or operations. Patients using myIBDcoach reported higher medication adherence rates $(P<0.001)$. myIBDcoach is therefore the best evaluated eHealth technology, but at present is specific to healthcare in the Netherlands. The impact on the IBD team compared to conventional care was that the gastroenterologist received less calls from patients in the myIBDcoach group as compared to the conventional care group ( 0.58 vs. 0.84 , $P=0.0003$ ) however calls to the specialist nurse were not significantly different between the groups ( 0.70 vs. 0.74 , $P=0.448)$.

\section{UCLA EIBD}

The University of California, Los Angeles (UCLA) Center for IBD has developed and eHealth technology that integrates PROs into eHealth records. This was launched by Daan Hommes in 2012 and can be found under "UCLA eIBD” in iTunes or Google Play stores, but full functionality is only available to patients treated at the UCLA Center for IBD. If a patient reaches a certain threshold of activity (as determined by the treating team), an automated message is sent to a nurse coordinator. ${ }^{27}$ A prospective randomized trial to assess the effect of this remote monitoring is awaited, but the goal is to improve disease control and as a consequence to reduce costs to payers and patients, potentially through lower insurance premiums if cared for by the UCLA Center for IBD.

\section{IBD Qorus}

IBD Qorus (http://www.crohnscolitisfoundation.org/ science-and-professionals/ibdqorus/) is the Crohn's Colitis Foundation of America national initiative to improve QoC for patients with IBD. ${ }^{28,29}$ As part of this initiative, a patientcentered tool has been developed. At this stage it is unclear which indices will be used however it will incorporate symptoms, QoL, self-management and shared decision-making between patient and provider. It aims to facilitate communication during and between visits, to improve QoC. It has been deployed in 30 U.S. hospitals and community practices, 
recruiting over 500 patients (Q4 2017). The initial metrics on QoC include the number of emergency department visits, anemia and nutrition, using an adaptation of the Malnutrition Universal Screening Tool scoring tool. It is proposed that when fully developed that the IBD Qorus platform will allow patients to look at their own data on a continuous basis. The appeal of IBD Qorus is that it is usable in daily U.S. practice whether a teaching hospital or community practice, having been carefully developed using PROMs that are very similar to those of ICHOM, to which the Crohn's and Colitis Foundation America and leading U.S. investigators also contributed. It has yet to demonstrate overall improvement in care, although favorable data are emerging at individual centers, allowing QoC to be compared and quality improvement of different interventions to be measured.

\section{TrueColours Ulcerative Colitis}

TrueColours Ulcerative Colitis is a comprehensive, webbased software package, housed on the National Health Service (NHS) server. ${ }^{30}$ Through email prompts linked to questionnaires, patients are able to enter daily symptoms (using the SCCAI), fortnightly QoL (using IBD Control-8, Crohn's Ulcerative Colitis Questionnaire-8, and EQ-5D), monthly home fecal calprotectin (using IBDoc ${ }^{\circledR}$ ) and 3-monthly ICHOM outcome measures. The system also allows blood pathology results, endoscopy (Ulcerative Colitis Endoscopic Index of Severity, UCEIS) and histopathology (Nancy Index) results to be collected. The TrueColours system, originally developed for bipolar disorder more than 10 years ago, uses a traffic light system to color-code responses. These thresholds were developed using previously published cutoffs. ${ }^{37}$

TrueColours Ulcerative Colitis was piloted in 66 patients for 6 months. Feasibility was demonstrated with $76 \%$ adherence to daily SCCAI and 95\% adherence to fortnightly QoL questionnaires, $75 \%$ adherence to monthly fecal calprotectin and $100 \%$ to 3 monthly outcomes measurements. Superior usability ratings were achieved with a median System Usability Score of 92.5 (IQR, 80-95). Qualitative, mixedmethods assessment using interviews found that patients considered TrueColours to be empowering, with increased levels of disease awareness, control and reassurance, as well as improved communication and decision making. Data from this small pilot have been used to create an index that predicts the need for escalation of therapy at a conventional outpatient visit with up to $95 \%$ accuracy, which may improve outpatient clinic resource utilization, mathematical analysis of different QoL indices, new fecal calprotectin cutoff levels that define disease remission based on endoscopy and histopathology (UCEIS and Nancy Indices). The TrueColours programme is currently being adapted to $\mathrm{CD}$ and is being rolled out to other NHS hospitals so that its impact on the IBD Service and potential for AI-directed management algorithms can be evaluated.

\section{CONCLUSIONS}

eHealth technology has the potential to improve IBD care. Remote monitoring of IBD, through patient reported outcomes, with direct connection to the IBD specialist team has been shown by some systems (myIBDcoach and ConstantCare among them) to enable more responsive and costeffective care.

Collecting outcomes' data on a large scale, most of which can be recorded by patients, allows comparisons in QoC between hospitals, as demonstrated by HealthPROMISE, IBD Qorus and myIBDcoach. It has to draw comparisons between regions or countries, independent of health care jurisdiction, so that quality improvement programmes can be appropriately focused.

As well as improving individual patient care and quality, eHealth technology is open to machine learning, which will not only allow analysis of data on a large scale but may allow future development of automated, personalized responses. IBD management teams need to embrace this technology.

\section{FINANCIAL SUPPORT}

The authors received no financial support for the research, authorship, and/or publication of this article.

\section{CONFLICT OF INTEREST}

No potential conflict of interest relevant to this article was reported.

\section{AUTHOR CONTRIBUTION}

Conceptualization: Simon Travis, Writing - original draft: Alissa Walsh. Writing - review and editing: Simon Travis, Approval of final manuscript: all authors. 


\section{REFERENCES}

1. Ng SC, Shi HY, Hamidi N, et al. Worldwide incidence and prevalence of inflammatory bowel disease in the 21 st century: a systematic review of population-based studies. Lancet 2018;390: 2769-2778.

2. van Deen WK, Choi JM, Zand A, et al. Sa1203 The development of e-Health tools for the management of inflammatory bowel diseases. Gastroenterology 2014;146(5 Suppl 1):S-229.

3. Rasmussen LM, Phanareth K, Nolte H, Backer V. Internet-based monitoring of asthma: a long-term, randomized clinical study of 300 asthmatic subjects. J Allergy Clin Immunol 2005;115:11371142.

4. Cox D, Ritterband L, Magee J, Clarke W, Gonder-Frederick L. Blood glucose awareness training delivered over the internet. Diabetes Care 2008;31:1527-1528.

5. Weinstock RS, Teresi JA, Goland R, et al. Glycemic control and health disparities in older ethnically diverse underserved adults with diabetes: five-year results from the Informatics for Diabetes Education and Telemedicine (IDEATel) study. Diabetes Care 2011;34:274-279.

6. Boyne JJ, Vrijhoef HJ, Wit Rd, Gorgels AP. Telemonitoring in patients with heart failure, the TEHAF study: study protocol of an ongoing prospective randomised trial. Int J Nurs Stud 2011;48: 94-99.

7. Boyne JJ, Van Asselt AD, Gorgels AP, et al. Cost-effectiveness analysis of telemonitoring versus usual care in patients with heart failure: the TEHAF-study. J Telemed Telecare 2013;19:242248.

8. Omboni S, Ferrari R. The role of telemedicine in hypertension management: focus on blood pressure telemonitoring. Curr Hypertens Rep 2015;17:535.

9. Kelly JJ, Sweigard KW, Shields K, Schneider D. Safety, effectiveness, and efficiency: a web-based virtual anticoagulation clinic. Jt Comm J Qual Saf 2003;29:646-651.

10. Basch E, Deal AM, Dueck AC, et al. Overall survival results of a trial assessing patient-reported outcomes for symptom monitoring during routine cancer treatment. JAMA 2017;318:197198.

11. Wootton R, Geissbuhler A, Jethwani K, et al. Comparative performance of seven long-running telemedicine networks delivering humanitarian services. J Telemed Telecare 2012;18:305311.

12. Sutherland D, Hayter M. Structured review: evaluating the effectiveness of nurse case managers in improving health outcomes in three major chronic diseases. J Clin Nurs 2009;18:2978-2992.
13. Kim AH, Roberts C, Feagan BG, et al. Developing a standard set of patient-centred outcomes for inflammatory bowel diseasean international, cross-disciplinary consensus. J Crohns Colitis 2018;12:408-418.

14. Coons SJ, Gwaltney CJ, Hays RD, et al. Recommendations on evidence needed to support measurement equivalence between electronic and paper-based patient-reported outcome (PRO) measures: ISPOR ePRO Good Research Practices Task Force report. Value Health 2009;12:419-429.

15. Taenzer P, Bultz BD, Carlson LE, et al. Impact of computerized quality of life screening on physician behaviour and patient satisfaction in lung cancer outpatients. Psychooncology 2000;9: 203-213.

16. Gutteling JJ, Busschbach JJ, de Man RA, Darlington AS. Logistic feasibility of health related quality of life measurement in clinical practice: results of a prospective study in a large population of chronic liver patients. Health Qual Life Outcomes 2008;6:97.

17. Erharter A, Giesinger J, Kemmler G, et al. Implementation of computer-based quality-of-life monitoring in brain tumor outpatients in routine clinical practice. J Pain Symptom Manage 2010;39:219-229.

18. Wright EP, Selby PJ, Crawford M, et al. Feasibility and compliance of automated measurement of quality of life in oncology practice. J Clin Oncol 2003;21:374-382.

19. Con D, De Cruz P. Mobile phone apps for inflammatory bowel disease self-management: a systematic assessment of content and tools. JMIR Mhealth Uhealth 2016;4:e13. doi: 10.2196/ mhealth.4874.

20. Kappelman MD, Palmer L, Boyle BM, Rubin DT. Quality of care in inflammatory bowel disease: a review and discussion. Inflamm Bowel Dis 2010;16:125-133.

21. Reddy SI, Friedman S, Telford JJ, Strate L, Ookubo R, Banks PA. Are patients with inflammatory bowel disease receiving optimal care? Am J Gastroenterol 2005;100:1357-1361.

22. Elkjaer M, Burisch J, Avnstrøm S, Lynge E, Munkholm P. Development of a web-based concept for patients with ulcerative colitis and 5-aminosalicylic acid treatment. Eur J Gastroenterol Hepatol 2010;22:695-704.

23. Cross RK, Cheevers N, Rustgi A, Langenberg P, Finkelstein J. Randomized, controlled trial of home telemanagement in patients with ulcerative colitis (UC HAT). Inflamm Bowel Dis 2012;18:1018-1025.

24. Cross RK, Cheevers N, Finkelstein J. Home telemanagement for patients with ulcerative colitis (UC HAT). Dig Dis Sci 2009;54: 2463-2472. 
25. Atreja A, Khan S, Rogers JD, et al. Impact of the mobile HealthPROMISE platform on the quality of care and quality of life in patients with inflammatory bowel disease: study protocol of a pragmatic randomized controlled trial. JMIR Res Protoc 2015; 4:e23. doi: 10.2196/resprot.4042.

26. de Jong M, van der Meulen-de Jong A, Romberg-Camps M, et al. Development and feasibility study of a telemedicine tool for all patients with IBD: myIBDcoach. Inflamm Bowel Dis 2017;23: 485-493.

27. Van Deen WK, van der Meulen-de Jong AE, Parekh NK, et al. Development and validation of an inflammatory bowel diseases monitoring index for use with mobile health technologies. Clin Gastroenterol Hepatol 2016;14:1742-1750.e7.

28. Hou J, Weatherly J, Sellin J, et al. P-132 Feasibility of screening for anemia using the Crohn's and colitis foundation anemia care pathway in IBD Qorus. Inflamm Bowel Dis 2017;23:S46S47.

29. Hou J, Xu A, Weatherly J, et al. P-129 CCFA quality of care breakthrough series collaborative: improving the delivery of urgent care for patients with IBD. Inflamm Bowel Dis 2017;23:S45-S46.

30. Walsh A. TrueColours: real time data collection in patients with ulcerative colitis [Doctoral dissertation]. University of Oxford, Oxford, UK; 2017.

31. Pedersen N, Elkjaer M, Duricova D, et al. eHealth: individualisation of infliximab treatment and disease course via a selfmanaged web-based solution in Crohn's disease. Aliment Pharmacol Ther 2012;36:840-849.
32. Pedersen N, Thielsen P, Martinsen L, et al. eHealth: individualization of mesalazine treatment through a self-managed webbased solution in mild-to-moderate ulcerative colitis. Inflamm Bowel Dis 2014;20:2276-2285.

33. Cross RK, Jambaulikar G, Langenberg P, et al. TELEmedicine for patients with inflammatory bowel disease (TELE-IBD): design and implementation of randomized clinical trial. Contemp Clin Trials 2015;42:132-144.

34. Atreja A, Khan S, Otobo E, et al. P554 Impact of real world home based remote monitoring on quality of care and quality of life in inflammatory bowel disease patients: one year results of pragmatic randomized trial. J Crohns Colitis 2017;11:S362-S363.

35. de Jong M, Van Der Meulen A, Romberg-Camps M, et al. Telemedicine enables a safe shift from examination room based care to personalized care for inflammatory bowel disease: a pragmatic randomized multicenter trial with myIBDcoach. Gastroenterology 2017;152(5 Suppl 1):S186.

36. Elkjaer M, Shuhaibar M, Burisch J, et al. E-health empowers patients with ulcerative colitis: a randomised controlled trial of the web-guided 'Constant-Care' approach. Gut 2010;59:1652-1661.

37. D'Haens G, Sandborn WJ, Feagan BG, et al. A review of activity indices and efficacy end points for clinical trials of medical therapy in adults with ulcerative colitis. Gastroenterology 2007;132: 763-786 